\title{
Acupuncture for persistent atrial fibrillation after catheter ablation: study protocol for a pilot randomized controlled trial
}

\author{
Ying Lin ${ }^{1}$, Xian Wang ${ }^{2}$, Xue-Bin Li ${ }^{3}$, Bang-Qi Wu ${ }^{4}$, Zhao-Hui Zhang ${ }^{5}$, Wei-Hua Guo ${ }^{6}$, Cun-Cao Wu ${ }^{3}$, Xin Chen \\ Ming-Long Chen ${ }^{8}$, Zhong Dai ${ }^{9}$, Fu-Yan Chen ${ }^{4}$, Rui Zhu ${ }^{5}$, Chu-Xi Liang ${ }^{9}$, Yun-Peng Tian ${ }^{7}$, Gang Yang ${ }^{8}$, \\ Chao-Qun Yan ${ }^{10}$, Jing Lu ${ }^{5}$, Hai-Ying Wang ${ }^{6}$, Jin-Ling Li', Jian-Feng Tu' ${ }^{11}$, He-Wen Li ${ }^{1}$, Dan-Dan Yang ${ }^{3}$, \\ Fang-Ting Yu ${ }^{1}$, Yu Wang ${ }^{1}$, Jing-Wen Yang ${ }^{1}$, Guang-Xia Shi ${ }^{1}$, Shi-Yan Yan ${ }^{1}$, Li-Qiong Wang ${ }^{1 *}$ (I) and Cun-Zhi Liu ${ }^{1}$
}

\begin{abstract}
Background: Atrial fibrillation (AF) is a common arrhythmia, which is closely related to cardiovascular morbidity and mortality. Although acupuncture is used in the treatment of AF, the evidence is insufficient. The objective of this pilot trial is to evaluate the feasibility, preliminary efficacy, and safety of acupuncture in reducing AF burden for persistent AF after catheter ablation (CA).

Methods and design: This will be a multi-center, 3-arm, pilot randomized controlled trial in China. Sixty patients in total will be randomly assigned to the specific acupoints group, the non-specific acupoints group, or the nonacupoints group in a 1:1:1 ratio. The whole study period is 6 months, including a 3-month treatment period and a 3-month follow-up period. All patients will receive 18 sessions of acupuncture over 12 weeks after CA and appropriate post-ablation routine treatment. The primary outcome is AF burden at 6 months after CA measured by electrocardiography patch that can carry out a 7-day continuous ambulatory electrocardiographic monitoring. The secondary outcomes include AF burden at 3 months after CA, recurrence of AF, quality of life, etc. The adverse events will also be recorded.
\end{abstract}

Discussion: This pilot study will contribute to evaluating the feasibility, preliminary efficacy, and safety of acupuncture in reducing AF burden for persistent AF after CA. The results will be used for the sample size calculation of a subsequent large-scale trial.

Trial registration: Chinese Clinical Trial Registry ChiCTR2000030576. Registered on 7 March 2020.

Keywords: Acupuncture, Atrial fibrillation burden, Catheter ablation, Persistent atrial fibrillation, Randomized controlled trial

\footnotetext{
* Correspondence: wangliqiongwork@163.com

${ }^{1}$ Acupuncture Research Center, School of Acupuncture-Moxibustion and

Tuina, Beijing University of Chinese Medicine, No. 11, Bei San Huan Dong Lu,

Chaoyang District, Beijing 100029, China

Full list of author information is available at the end of the article
}

(c) The Author(s). 2021 Open Access This article is licensed under a Creative Commons Attribution 4.0 International License, which permits use, sharing, adaptation, distribution and reproduction in any medium or format, as long as you give appropriate credit to the original author(s) and the source, provide a link to the Creative Commons licence, and indicate if changes were made. The images or other third party material in this article are included in the article's Creative Commons licence, unless indicated otherwise in a credit line to the material. If material is not included in the article's Creative Commons licence and your intended use is not permitted by statutory regulation or exceeds the permitted use, you will need to obtain permission directly from the copyright holder. To view a copy of this licence, visit http://creativecommons.org/licenses/by/4.0/ The Creative Commons Public Domain Dedication waiver (http://creativecommons.org/publicdomain/zero/1.0/) applies to the data made available in this article, unless otherwise stated in a credit line to the data. 


\section{Background}

Atrial fibrillation (AF), as a common arrhythmia, increases in prevalence with advancing age [1]. Patients with AF have a 4-5 times greater risk of ischemic stroke than non-AF patients [2], resulting in $20 \%$ mortality and $60 \%$ disability when a stroke occurs [3]. AF triples the risk of heart failure [4] and doubles the risk of myocardial infarction [5]. In addition, AF is associated with a decreased quality of life and depression [6].

Catheter ablation $(\mathrm{CA})$ is an invasive procedure used to treat AF that uses radiofrequency or other energy sources to isolate portions of the left atrium, such as the pulmonary veins, and other areas. For paroxysmal AF, although outcomes have improved over the last 20 years, success rates of CA, defined as freedom from recurrent AF, have plateaued in the $60-70 \%$ range [7]. For persistent $\mathrm{AF}$, success rates are even more unfavorable at 45 to $60 \%$ at 1 year $[8,9]$. There remain unmet needs in improving the ability to restore and sustain sinus rhythm [6]. Acupuncture, as a nonpharmacologic therapy, has been widely applied in cardiovascular diseases [10-12]. Acupuncture plays a role in regulating the autonomic nervous system in the treatment of many diseases [1315]. The prevention effect of acupuncture on the recurrence of AF may have been achieved in this way. Previous trials have indicated that acupuncture could prevent persistent AF recurrence after electrical cardioversion or CA $[16,17]$. However, in these trials, only the recurrence rate was evaluated, and there was no quantitative index like AF burden (the percentage of time in AF during a monitoring period) [18] and quality of life assessment was overlooked. Through a relatively simple detection method, the recurrence rate may be underestimated. Both are single-center studies, which could result in bias. As for the control setting, the blinding method cannot be completely achieved and the accurate efficacy of acupuncture was unable to be measured in the studies. Therefore, it is difficult to draw sound conclusions from the results of these trials due to the existing limitations of their design. In this context, we designed a multicenter, placebo-controlled pilot randomized trial to evaluate the feasibility, preliminary efficacy, and safety of acupuncture. AF burden will be the primary outcome and the recurrence rate will be measured by a relatively superior detection method.

It is well known that the efficacy of acupuncture is related to the acupoints selected. Some acupoints are called specific acupoints in traditional Chinese medicine (TCM), which have special properties, therapeutic effects, and a specific name $[19,20]$. Specific acupoints have been applied in the treatment of many diseases $[10$, $21,22]$, which are believed to be a group of acupoints superior to others in treating specific diseases. The aim of our study is as follows: (i) to explore the feasibility and provide power calculations for a future large-scale clinical trial, (ii) to explore the efficacy and safety of acupuncture as adjunctive therapy of routine care in reducing AF burden among patients with persistent AF after $\mathrm{CA}$, and (iii) to examine whether the specific acupoints are superior to either non-specific acupoints or nonacupoints in therapeutic efficacy.

\section{Methods/design \\ Study design}

This multi-center, 3-arm, assessor and statistician blinded, pilot randomized controlled trial (RCT) will be conducted at four centers in China: Dongzhimen Hospital Affiliated to Beijing University of Chinese Medicine; Peking University People's Hospital; The First Affiliated Hospital of Nanjing Medical University; Tianjin University of Traditional Chinese Medicine. Eligible patients will be enrolled from inpatient units of the cardiology departments at these hospitals. The whole study period will be 6 months and consist of a 3-month treatment period and a 3-month follow-up period. The CONSORT statement [23] and STRICTA guidelines [24] for acupuncture studies have been used as a framework for the methodology in this study. Besides, this protocol is reported following the SPIRIT guidelines (Additional file 1) [25]. The flowchart of this study is shown in Fig. 1.

\section{Participants}

Patients with persistent AF will be diagnosed according to the 2016 ESC guidelines [6]. Persistent AF is defined as AF that lasts for more than 7 days, including episodes that are terminated by cardioversion after 7 days or more [6].

\section{Inclusion criteria}

1. Persistent AF lasting no more than 3 years.

2. Age between 18 and 75 years old (male or female).

3. No acupuncture treatment within the previous 6 months.

\section{Exclusion criteria}

1. Severe heart failure (New York Heart Association class III or IV).

2. Echocardiographic parameters: left ventricular ejection fraction $<40 \%$, left atrium diameter $>5.0 \mathrm{~cm}$.

3. Severe lung, liver, kidney disease, or other serious primary diseases.

4. Skin allergy to electrode patches for electrocardiographic monitoring.

5. The estimated life expectancy of $<1$ year. 


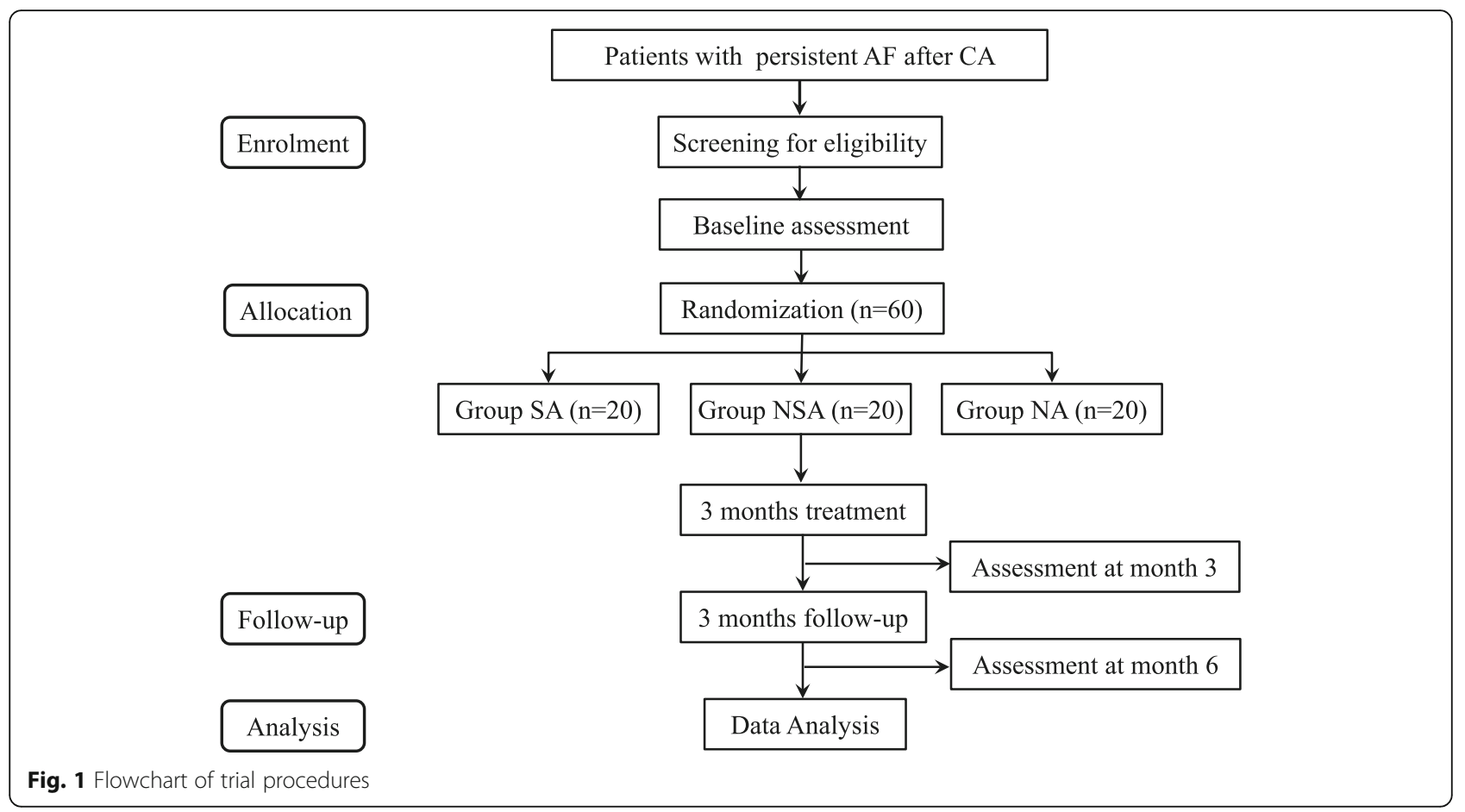

\section{Ablation procedure}

Physicians who perform ablations are required to have done 100 cases prior to this trial. All centers will cooperate with a relatively uniform standard of ablation procedures. All antiarrhythmic drugs (AADs), except amiodarone, will be discontinued for at least five halflives before the procedure. Amiodarone will be recommended to be discontinued 3 months before the ablation procedure. Warfarin or novel oral anticoagulants should be taken for at least 3 weeks before CA. Transesophageal echocardiography will be performed on the day of CA or 1 day before CA to rule out left atrial thrombus.

All patients will undergo pulmonary vein isolation (PVI) by radiofrequency ablation. Selection of the type of ablation catheter, settings of power and irrigation, and the use of 3-dimensional mapping systems will be at the discretion of the physicians performing CA. It is regarded as an endpoint that the complete entrance and exit block of all pulmonary vein antra are confirmed in PVI. If the patient is still in a state of AF after PVI, electrical cardioversion will be performed. Patients who fail to be converted to sinus rhythm after PVI or electrical cardioversion will be excluded from this study.

\section{Randomization and blinding}

All the patients will be randomly assigned (in a 1:1:1 ratio) to the specific acupoints (SA) group, non-specific acupoints (NSA) group, or non-acupoints (NA) group. NA group is the control group in this trial. Stratification will be performed by center and the method of block randomization will be applied in each center. An independent statistician who is not involved in treatment and outcome assessment will be in charge of computergenerated randomization sequence using SAS 9.3 software (SAS Institute, Cary, NC, USA). The allocation schedule will be carried out by an independent researcher who will be responsible for providing random numbers and group assignments to recruiters at each center through a centralized telephone randomization procedure. The patients, outcome assessors, and statisticians will be blinded. However, the acupuncturists will not be blinded due to the nature of acupuncture. The allocations will not be revealed until the data's statistical analysis is completed.

\section{Interventions}

The acupuncture regimens were determined according to the clinical practice of acupuncture experts and literature on acupuncture treatment of AF $[16,17,26,27]$. All acupuncture operations will be performed by professional acupuncturists who have obtained a Chinese medicine practitioner license with more than 3 years of clinical experience. Besides, all patients will receive relatively uniform post-ablation management after CA.

Before the study, all acupuncturists will be specially trained in the standardized operation procedure of acupuncture, including accurate positioning of acupoints, operational methods, and so on. Besides, brochures 
showing the information on the standardized operation in detail will be provided to the acupuncturists for reference. Within 3 months after CA (blanking period), patients will receive acupuncture treatment twice per week for weeks 1-6 and once per week for weeks 7-12 (18 sessions in total). Sterilized disposable steel needles (Hwato disposable acupuncture needle; Suzhou, Jiangsu, China) will be used in the treatment and in sizes of 0.30 $\mathrm{mm} \times 25 \mathrm{~mm}$ or $0.30 \mathrm{~mm} \times 40 \mathrm{~mm}$. Standardized prescriptions will be used in the three groups. Bilateral xinshu (BL15), neiguan (PC6), and shenmen (HT7) will be selected in the SA group. Bilateral pohu (BL42), tianquan (PC2), and qingling (HT2) will be selected in the NSA group. The code and location of the acupoints are consistent with the WHO standards [28]. The exact locations of all acupoints are listed in Table 1 and marked in Fig. 2. The three non-acupoints chosen for the NA group are separated from known acupoints. The locations of non-acupoints are shown in Table 2 and also marked in Fig. 2.

\section{SA group}

All four acupoints in this group are specific acupoints. Acupuncture is performed in a certain sequence of acupoints: BL15, PC6, and HT7. Firstly, BL15 will be punctured obliquely to a depth of 1.0$1.6 \mathrm{~cm}$. Then, the needles will be stimulated (lift and thrust or twirl and rotate the needles) manually to achieve the Deqi sensation (the sensation including soreness, numbness, distention, or heaviness accompanied by acupuncture). The stimulation will continue for $1 \mathrm{~min}$, and then the needles will be pulled out. Secondly, PC6 will be punctured perpendicularly 1.0$2.0 \mathrm{~cm}$. HT7 will be punctured perpendicularly 0.6$1.0 \mathrm{~cm}$. PC6 and HT7 are also stimulated to achieve Deqi. After that, needles will be retained in place for $30 \mathrm{~min}$.

\section{NSA group}

Non-specific acupoints are a group of acupoints that are not thought to have similar specific effects to specific acupoints. The nearby non-specific points belonging to the same meridians of the specific acupoints group for treatment were selected as in some previous studies [21, 22]. The sequence of acupuncture will be BL42, PC2, and HT2. BL42 will be punctured obliquely $1.0-1.6 \mathrm{~cm}$ using identical operation with BL15. PC2 will be punctured perpendicularly $2.0-3.0 \mathrm{~cm}$. HT2 will be punctured perpendicularly $1.0-2.0 \mathrm{~cm}$. The acupuncturist will use the same operation as PC6 and HT7 at PC2 and HT2 in the SA group.

\section{NA group}

Shallow puncture $(2-3 \mathrm{~mm}$ in depth) will be performed without obtaining Deqi. It will be safe and not do any harm to the patients. Acupuncture will follow this sequence: NA1, NA2, NA3. Needles in NA2 and NA3 should be left for $30 \mathrm{~min}$, whereas needles will not be retained in NA1.

\section{Post-ablation management}

Electrical cardioversion is allowed within 3 months after CA (blanking period). The blanking period refers to a period of 3 months after any form of cardiac ablation during which arrhythmias are not considered. Patients who experience AF recurrence after the blanking period will be allowed to start or resume AADs or undergo a repeat ablation. According to the guidelines of the ESC [6] and Chinese guidelines [29] for the management of $\mathrm{AF}$, all patients will receive routine medication after CA. The drugs protocol includes anticoagulation (at least 8 weeks after CA), AADs (continued 3 months after CA), and proton pump inhibitor (4 weeks). Moreover, patients are required to keep a detailed record of their medication use, including name, duration, and dose.

Table 1 Locations of acupoints for specific acupoints group and non-specific acupoints group

\begin{tabular}{|c|c|c|}
\hline & Acupoint & Location \\
\hline \multirow[t]{3}{*}{ Specific acupoints } & Xinshu (BL15) & $\begin{array}{l}\text { In the upper back region, at the same level as the inferior border of the spinous process of } \\
\text { the fifth thoracic vertebra (T5), } 1.5 \mathrm{cun}^{\mathrm{a}} \text { lateral to the posterior median line. }\end{array}$ \\
\hline & Neiguan (PC6) & $\begin{array}{l}\text { On the anterior aspect of the forearm, between the tendons of the palmaris longus and the } \\
\text { flexor carpi radialis, } 2 \text { cun proximal to the palmar wrist crease. }\end{array}$ \\
\hline & Shenmen (HT7) & $\begin{array}{l}\text { On the anteromedial aspect of the wrist, radial to the flexor carpi ulnaris tendon, on the palmar } \\
\text { wrist crease. }\end{array}$ \\
\hline \multirow[t]{3}{*}{ Non-specific acupoints } & Pohu (BL42) & $\begin{array}{l}\text { In the upper back region, at the same level as the inferior border of the spinous process of } \\
\text { the third thoracic vertebra (T3), } 3 \text { cun lateral to the posterior median line. }\end{array}$ \\
\hline & Tianquan (PC2) & $\begin{array}{l}\text { On the anterior aspect of the arm, between the long head and short head of the biceps } \\
\text { brachii muscle, } 2 \text { cun distal to the anterior axillary fold. }\end{array}$ \\
\hline & Qingling (HT2) & $\begin{array}{l}\text { On the medial aspect of the arm, just medial to the biceps brachii muscle, } 3 \text { cun superior to } \\
\text { the cubital crease. }\end{array}$ \\
\hline
\end{tabular}

${ }^{\mathrm{a}} 1$ cun $(\approx 20 \mathrm{~mm})$ is defined as the width of the interphalangeal joint of the patient's thumb 


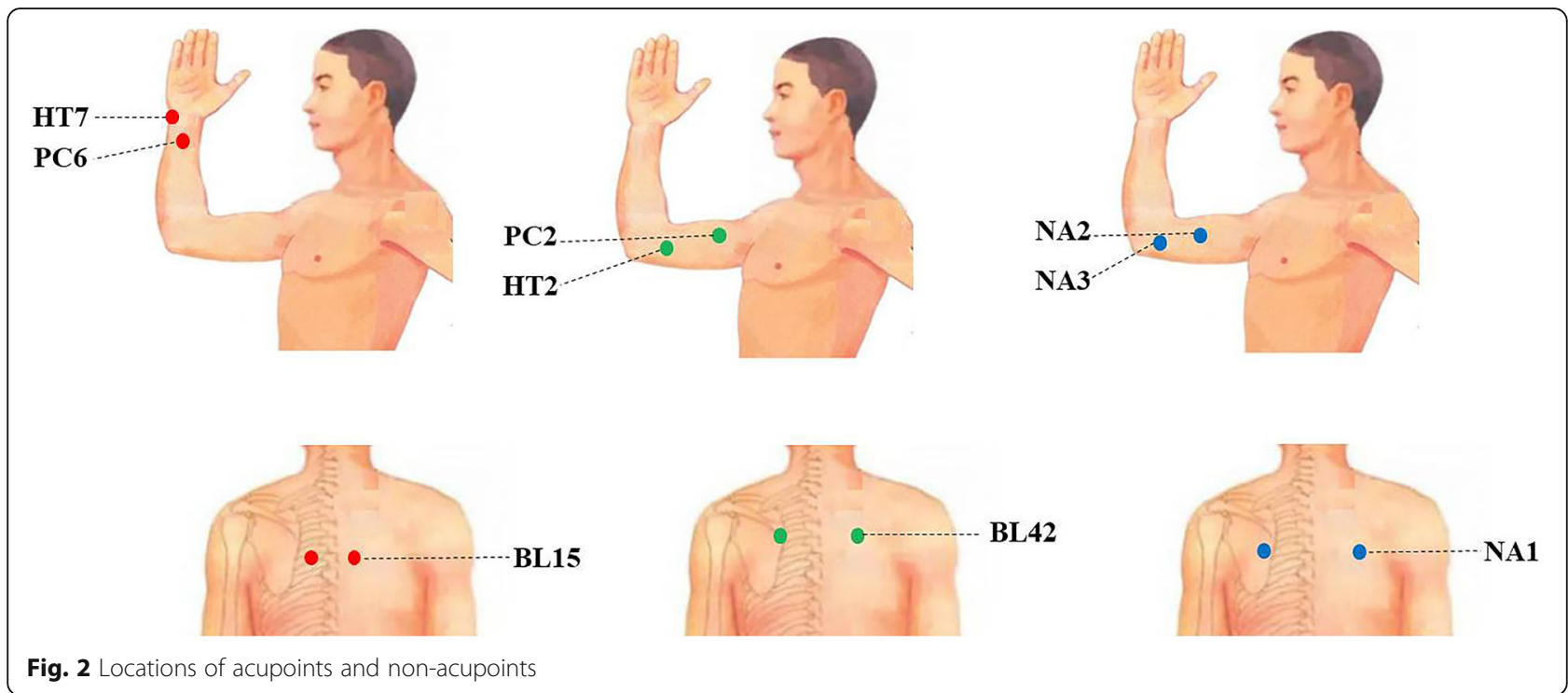

\section{Outcomes}

\section{Primary outcome measurement}

The primary outcome is the AF burden at 6 months after CA. AF burden will be measured by NS-SP-B-01 smart patch (Ensense Biomedical Technologies Co., Ltd., Shanghai, China). The smart patch will carry out continuous ambulatory electrocardiographic monitoring for 7 days. AF burden is expressed in the form of a percentage, which specifically refers to the proportion of time in AF within 7-days' long for one patient in this study (range $0-100 \%$ ). When the monitoring is complete, the patient will return the smart patch and the researchers can get the recordings. After the researcher transmits the recordings to the Ensense Company, the recordings will be analyzed by an appropriate algorithm. The results then will be reviewed by certified cardiac technicians to assure analysis quality and generate a report transmitted to the researchers.

\section{Secondary outcome measurements}

AF burden at 3 months after CA AF burden at 3 months after CA will be measured by the same method as the primary outcome. This outcome is to evaluate whether acupuncture can reduce the duration of $\mathrm{AF}$ after treatment.

Table 2 Locations of non-acupoints group

\begin{tabular}{ll}
\hline Non-acupoints & Location \\
\hline NA1 & $\begin{array}{l}\text { In the middle of Shentang (BL44) and Tianzong } \\
(\mathrm{SI11}) \text { points }\end{array}$ \\
NA2 & $\begin{array}{l}\text { In the middle of Tianquan (PC2) and Qingling } \\
(\mathrm{HT2}) \text { points }\end{array}$ \\
NA3 & $\begin{array}{l}\text { In the middle of Quze (PC3) and Qingling } \\
(\mathrm{HT} 2) \text { points }\end{array}$ \\
\hline
\end{tabular}

Time to AF recurrence Recurrence of AF within 6 months after CA will be measured, and it is defined as any atrial tachyarrhythmia including AF/ atrial flutter/ atrial tachycardia lasting $30 \mathrm{~s}$ or longer after the 3month blanking period. Patients will be instructed to obtain 12-lead electrocardiograph (ECG) or 24 h Holter in order to verify the recurrence whenever they experience AF-related symptoms (palpitations, dyspnea, chest pain, etc.). Additionally, recurrence will be monitored via the smart patch regardless of the existence of symptoms at the end of 3 and 6 months. All researchers are required to receive training in the procedure of electrocardiographic monitoring and the use of the NS-SP-B-01 smart patch. They will then be responsible for explaining the use of the device to the patients.

Atrial Fibrillation Effect and Quality of Life Scale (AFEQT) The AFEQT [30] is a questionnaire specially designed to assess the quality of life in atrial fibrillation patients. The assessment will take place at baseline and 3 and 6 months. A summary score and subscale scores in the following 3 domains: symptoms, daily activities, and treatment will be calculated. Summary and subscale scores range from 0 to 100 (complete to no AF-related disability).

Heart rate variability Heart rate variability will be generated while monitoring AF burden with the smart patch to quantitatively evaluate the balance between the sympathetic and vagus nerves at 3 and 6 months.

Credibility and expectancy A Credibility/Expectancy Questionnaire [31] will be used to measure the patients' attitudes toward acupuncture before treatment. 
Blinding assessment Blind evaluation will be conducted in order to evaluate whether the blind method is successful. After treatments in weeks 6 and 12, all patients will be asked to guess whether the needles go deep or shallow layers in the acupuncture treatment they receive. Before the treatment, one or two doctors of each center will be in charge of recruiting the patients and gaining informed consent from them. The patients will be informed: "There are three different groups of acupuncture with three different acupoint selection schemes. You are equally likely to be assigned to all three groups, and previous studies have shown that all three methods were associated with positive outcomes in clinical studies."

Others They include the number of electrical cardioversion within the blanking period, the number of repeat $\mathrm{CA}$ after the blanking period, and the number of AFrelated hospitalizations.

\section{Adverse events}

Any adverse events and how they are dealt with will be recorded from baseline to 6 months. These adverse events specifically included bleeding, hematoma, fainting, severe pain, and local infection. If any serious adverse event occurs to the patient, which will be immediately reported to the primary investigator and all details will be recorded. The patient will be withdrawn from the trial.

\section{Data management}

Detailed time points of outcome assessments are provided in Fig. 3. Throughout this study, the data of all patients will be recorded on the case report form (CRF). When the forms are completed, the data will be entered into the Excel spreadsheet independently by two researchers. There will be a data manager to compare the two sets of data. If any differences are found, they will be corrected according to the original CRFs. All documents related to the research, including the paper files and electronic documents, will be well preserved for no

\begin{tabular}{|c|c|c|c|c|c|c|}
\hline \multirow[b]{3}{*}{ TIME POINT } & \multicolumn{6}{|c|}{ Study period } \\
\hline & \multirow{2}{*}{$\begin{array}{c}\text { Enrollment } \\
\text { Day -3 }\end{array}$} & \multirow{2}{*}{$\begin{array}{c}\text { Allocation } \\
\mathbf{0}\end{array}$} & \multicolumn{3}{|c|}{ Post- allocation } & \multirow{2}{*}{\begin{tabular}{|l|} 
Closeout \\
Month 6 \\
\end{tabular}} \\
\hline & & & $\mathbf{0}$ & Week 6 & Month 3 & \\
\hline \multicolumn{7}{|l|}{ ENROLLMENT: } \\
\hline \multirow{4}{*}{$\begin{array}{l}\text { Eligibility screen } \\
\text { Informed consent } \\
\text { Randomization } \\
\text { Allocation }\end{array}$} & $x$ & & & & & \\
\hline & $x$ & & & & & \\
\hline & $x$ & & & & & \\
\hline & & $x$ & & & & \\
\hline \multirow{4}{*}{$\begin{array}{l}\text { INTERVENTION: } \\
\text { Specific acupoints } \\
\text { Non-specific acupoints } \\
\text { Non-acupoints }\end{array}$} & & & & & & \\
\hline & & & $\leftarrow$ & & $\longrightarrow$ & \\
\hline & & & $\leftarrow$ & & $\longrightarrow$ & \\
\hline & & & $\leftarrow$ & & $\longrightarrow$ & \\
\hline \multicolumn{7}{|l|}{ ASSESSMENT: } \\
\hline \multirow{5}{*}{$\begin{array}{l}\text { AF burden } \\
\text { AF recurrence } \\
\text { AFEQT } \\
\text { Heart rate variability } \\
\text { Blinding ascecsment }\end{array}$} & & & & & $x$ & $x$ \\
\hline & & & & & $x$ & $x$ \\
\hline & & & $x$ & & $x$ & $x$ \\
\hline & & & & & $x$ & $x$ \\
\hline & & & & $x$ & $x$ & \\
\hline \multirow{2}{*}{$\begin{array}{l}\text { Blinding assessment } \\
\text { Credibility/Expectancy } \\
\text { Electrical cardioversion }\end{array}$} & & & $x$ & & & \\
\hline & & & $x$ & $x$ & $x$ & \\
\hline \multirow{2}{*}{$\begin{array}{l}\text { Repeat ablation } \\
\text { AF-related } \\
\text { hospitalizations }\end{array}$} & & & & & $x$ & $x$ \\
\hline & & & $x$ & $x$ & $x$ & $x$ \\
\hline Adverse events & & & $x$ & $x$ & $x$ & $x$ \\
\hline
\end{tabular}

Fig. 3 Schedule of enrollment, intervention, and assessments (SPIRIT figure). AF, atrial fibrillation; AFEQT, Atrial Fibrillation Effect on Quality of Life 
less than 5 years after publication. The first author or corresponding author will provide the original data if anyone puts forward questions about the published data. It will be protected and never be disclosed that the patients' personal privacy information includes name, gender, age, address, and telephone number. The ethics committee of Beijing University of Chinese medicine will audit trial conduct every 12 months.

\section{Sample size}

The purpose of this pilot study is to evaluate the efficacy and safety of acupuncture in the treatment of AF and the feasibility for a further large-scale clinical trial. We did not find a suitable reference to calculate the sample size, but a minimum sample size of the clinical trial with 20 patients per group is used instead [32]. A total of 60 patients will be randomly assigned equally to SA, NSA, or NA group. This trial will provide data for sample size calculations of a future large-scale RCT.

\section{Statistical analysis}

Baseline characteristics will be summarized by the treatment group. Continuous variables will be described as mean \pm standard deviations (normal distribution), or medians and interquartile ranges (non-normal distributions). Analysis of variance (ANOVA) or Kruskal-Wallis test (if normality is violated) will be applied in the comparison among three groups. Categorical variables will be described as frequency (percentage) and compared by $\chi^{2}$ test or Fisher's exact test etc. As for the primary outcome, AF burden is expressed in the form of a percentage, which specifically refers to the proportion of time in AF within 7-days' long for one patient in this study (range 0-100\%). The null hypothesis that AF burden would be equivalent among the three arms (H0: effect of $\mathrm{SA}=$ effect of NSA = effect of NA) will be tested. The means of AF burden at 6 months of the three arms will be compared using ANOVA based on the preliminary assessment of the data distributions. If the ANOVA test among 3 arms is significant, the pairwise comparison will be performed by the least significant difference (LSD) test, and mean differences of pairwise comparison will be reported as effect measure. If AF burden is a skewed distribution, the data will be transformed to a normal distribution by corresponding methods for further comparison, and the same statistical analysis strategy will be adopted. The comparisons of AF recurrence between the three arms at 6 months will be performed based on a time-to-first-event analysis using the logrank test. Kaplan-Meier cumulative AF recurrence rates will be calculated for each arm, with event or censoring times measured from the time of randomization. Hazard ratios (HRs) with associated 95\% CIs will be derived using the Cox proportional hazards model.
All efficacy analyses will be based on the intention-totreat principle. Missing data on the primary outcomes will be imputed by multiple imputations. An independent statistician who is blinded will conduct the statistical analysis using SAS 9.3. We will set a significance level of $P<0.05$ (two-sided) without adjustment on account of the values considered explorative.

\section{Ethics and dissemination}

The trial will be conducted in accordance with the Declaration of Helsinki and has been approved by the Ethics Committee of Beijing University of Chinese Medicine (No. 2020BZHYLL0106). Patients will be included only after the details of the study explained to them and signing informed consent forms. The results of the trial will be published in a peer-reviewed academic journal.

\section{Discussion}

AF is one of the leading causes of stroke and cardiovascular diseases. It is of great significance to find combination therapy to maintain sinus rhythm. This pilot study is designed to evaluate the feasibility, efficacy, and safety of acupuncture in reducing AF burden in a specific group of patients with persistent AF after CA.

Previously, an RCT was conducted to prevent AF recurrence after electrical cardioversion with acupuncture in persistent AF [16]. AF was detected only by 12-lead ECG, which could underestimate the recurrence rate of AF. Another study was designed to determine whether acupuncture could reduce the early recurrences of AF after $\mathrm{CA}$ in persistent AF [17]. In the study, the measured recurrence rate of AF was the early recurrence rate within the blanking period, and the long-term prognosis was not studied, which is a more appropriate endpoint. In addition, neither of the two studies evaluated the quality of life that is of great concern in AF patients $[33,34]$.

The recurrence rate is often used as the primary outcome in clinical trials on AF. However, recurrence is a binary concept, reflecting only the presence and absence. In contrast, AF burden is the primary outcome of our study, which can represent the quantity or amount of AF that one person has. It helps analyze the therapeutic efficacy quantitatively. The AF recurrence after the blanking period will be set as a significant secondary outcome in our study. We will both use 12-lead ECG and 24-h Holter during the monitoring, which may ensure AF recurrence detected in time. Moreover, we will evaluate the quality of life through AFEQT. The control group is the NA group in which shallow insertion at non-acupoint is a kind of sham treatment of acupuncture. Shallow insertion in non-acupoints applied as a sham control was established in the light of two literature reviews $[35,36]$. In addition, this placebo control 
has been verified to be successful in blinding Chinese patients suffering from postprandial distress syndrome [37] and chronic severe functional constipation [38]. Compared with a blank control group, sham acupuncture can largely eliminate the interference of nonspecific effects. We will choose the acupoints selection scheme with better efficacy through the comparison between group SA and group NSA, which will be applied in the future large-scale RCT.

There are some limitations to our study. First, we will not be able to monitor AF at all the times by using implant loop recorder, since very few patients are willing to do invasive monitoring. Second, as a pilot study, the sample size is small, and it is impossible to evaluate the long-term efficacy without insufficient follow-up time in this study. Nevertheless, after that, we will conduct a large-scale RCT to solve these problems. Third, acupuncturists cannot be blinded during acupuncture owing to the nature of the intervention, which may generate bias.

\section{Trial status}

This trial is currently recruiting patients.

\section{Supplementary Information}

Supplementary information accompanies this paper at https://doi.org/10. 1186/s13063-020-04967-y.

Additional file 1. Completed Standard Protocol Items: Recommendation for Interventional Trials (SPIRIT) 2013 Checklist: items addressed in this clinical trial protocol.

Additional file 2. STRICTA 2010 checklist of information to include when reporting interventions in a clinical trial of acupuncture (Expansion of Item 5 from CONSORT 2010 checklist).

\section{Abbreviations}

AF: Atrial fibrillation; CA: Catheter ablation; TCM: Traditional Chinese medicine; RCT: Randomized controlled trial; PVI: Pulmonary vein isolation; AADs: Antiarrhythmic drugs; SA: Specific acupoints; NSA: Non-specific acupoints; NA: Non-acupoints; ECG: Electrocardiograph; AFEQT: Atrial Fibrillation Effect and Quality of Life Scale; CRF: Case report form; ANOVA: Analysis of variance; LSD: Least significant difference; HRs: Hazard ratios

\section{Acknowledgements}

We are grateful to Prof. Mintu Turakhia (Stanford University) for his help in study design and manuscript modification. We would like to thank Prof. Marc Fisher (Harvard University) for revising the manuscript. Besides, the authors would like to express their sincere thanks to all patients participating in the trial.

\section{Authors' contributions}

CZL conceived the study. CZL, LQW, YL, and JLL initiated the study design. LQW, JFT, and SYY drew up the statistical plan. XW, XBL, BQW, ZHZ, WHG, $X C, M L C, Z D, F Y C, R Z, C X L, Y P T, G Y, H Y W$, JLL, HWL, DDY, FTY, and YW helped with its implementation. YL, LQW, JWY, GXS, and CZL drafted and critically revised the manuscript for important intellectual content. CZL sought funding. LQW, CCW, JL, BQW, and CQY sought ethical approval. All authors contributed to the refinement of the study protocol and approved the final manuscript.

\section{Funding}

This study has been supported by the National Key Research and Development Program of China (2019YFC1712102). The project was funded by Ministry of Science and Technology of the People's Republic of China (the address: 5B, Fuxing Road, Beijing, 100862, P.R. China). New teachers start fund project of Beijing University of Chinese Medicine also have funded our research. The funder has no role in the design of the trial; collection, management, analysis, and interpretation of data; and writing of the report or the decision to submit the report for publication. During the whole research process, the funder will provide funds for the research and regularly inspect the progress of the study.

\section{Availability of data and materials}

All individual participants' data collected during the trial will be available for anyone who wishes to access the data immediately following publication in accordance with FAIR principles.

\section{Ethics approval and consent to participate}

The study protocol has been approved by the Ethics Committee of Beijing University of Chinese Medicine (Approval Number: 2020BZHYLL0106). Written informed consent will be obtained from all patients before enrolment.

\section{Consent for publication}

Not applicable.

\section{Competing interests}

None declared.

\section{Author details}

${ }^{1}$ Acupuncture Research Center, School of Acupuncture-Moxibustion and Tuina, Beijing University of Chinese Medicine, No. 11, Bei San Huan Dong Lu, Chaoyang District, Beijing 100029, China. ${ }^{2}$ Dongzhimen Hospital Affiliated to Beijing University of Chinese Medicine, Beijing University Cardiology Research Institute of Traditional Chinese Medicine, Beijing 100700, China. ${ }^{3}$ Department of Cardiology, Peking University People's Hospital, Beijing 100044, China. ${ }^{4}$ National Clinical Research Center for Chinese Medicine Acupuncture and Moxibustion, First Teaching Hospital, Tianjin University of Traditional Chinese Medicine, Tianjin 300193, China. ${ }^{5}$ Department of Acupuncture and Moxibustion, The First Affiliated Hospital of Nanjing Medical University, Nanjing 210029, China. 'Department of Cardiology, Dongzhimen Hospital Affiliated to Beijing University of Chinese Medicine, Beijing 100700, China. ${ }^{7}$ Department of Cardiology, Tianjin First Center Hospital, Tianjin 300192, China. ${ }^{8}$ Department of Cardiology, The First Affiliated Hospital of Nanjing Medical University, Nanjing 210029, China. ${ }^{9}$ Department of Traditional Chinese Medicine, Peking University People's Hospital, Beijing 100044, China. ${ }^{10}$ Department of Acupuncture and Moxibustion, Dongzhimen Hospital Affiliated to Beijing University of Chinese Medicine, Beijing 100700, China.

${ }^{11}$ Department of Acupuncture and Moxibustion, Beijing Hospital of Traditional Chinese Medicine Affiliated to Capital Medical University, Beijing 100010, China.

Received: 18 May 2020 Accepted: 11 December 2020

Published online: 07 January 2021

\section{References}

1. Wolf PA, Benjamin EJ, Belanger AJ, Kannel WB, Levy D, D'Agostino RB. Secular trends in the prevalence of atrial fibrillation: the Framingham study. Am Heart J. 1996;131(4):790-5.

2. Chien K, Su T, Hsu H, Chang W, Chen P, Chen M, et al. Atrial fibrillation prevalence, incidence and risk of stroke and all-cause death among Chinese. Int J Cardiol. 2010;139(2):173-80.

3. Gladstone DJ, Bui E, Fang J, Laupacis A, Lindsay MP, Tu JV, et al. Potentially preventable strokes in high-risk patients with atrial fibrillation who are not adequately anticoagulated. Stroke. 2009;40(1):235-40.

4. Morin DP, Bernard ML, Madias C, Rogers PA, Thihalolipavan S, Estes NR. The state of the art: atrial fibrillation epidemiology, prevention, and treatment. Mayo Clin Proc. 2016;91(12):1778-810.

5. Soliman EZ, Safford MM, Muntner P, Khodneva Y, Dawood FZ, Zakai NA, et al. Atrial fibrillation and the risk of myocardial infarction. JAMA Intern Med. 2014;174(1):107. 
6. Kirchhof P, Benussi S, Kotecha D, Ahlsson A, Atar D, Casadei B, et al. 2016 ESC Guidelines for the management of atrial fibrillation developed in collaboration with EACTS. Eur Heart J. 2016;37(38):2893-962.

7. Perino AC, Leef GC, Cluckey A, Yunus FN, Askari M, Heidenreich PA, et al. Secular trends in success rate of catheter ablation for atrial fibrillation: the SMASH-AF cohort. Am Heart J. 2019;208:110-9.

8. Verma A, Jiang CY, Betts TR, Chen J, Deisenhofer I, Mantovan R, et al. Approaches to catheter ablation for persistent atrial fibrillation. N Engl J Med. 2015;372(19):1812-22

9. Vogler J, Willems S, Sultan A, Schreiber D, Luker J, Servatius H, et al. Pulmonary vein isolation versus defragmentation: the CHASE-AF clinical trial. J Am Coll Cardiol. 2015;66(24):2743-52.

10. Zhao L, Li D, Zheng H, Chang X, Cui J, Wang R, et al. Acupuncture as adjunctive therapy for chronic stable angina. JAMA Intern Med. 2019; 179(10):1388.

11. Wang Q, Liang D, Wang F, Li W, Han Y, Zhang W, et al. Efficacy of electroacupuncture pretreatment for myocardial injury in patients undergoing percutaneous coronary intervention: a randomized clinical trial with a 2-year follow-up. Int J Cardiol. 2015;194:28-35.

12. Mehta PK, Polk DM, Zhang X, Li N, Painovich J, Kothawade K, et al. A randomized controlled trial of acupuncture in stable ischemic heart disease patients. Int J Cardiol. 2014;176(2):367-74.

13. Benrick A, Kokosar M, Hu M, Larsson M, Maliqueo M, Marcondes RR, et al. Autonomic nervous system activation mediates the increase in whole-body glucose uptake in response to electroacupuncture. FASEB J. 2017;31(8): 3288-97.

14. Backer M, Grossman P, Schneider J, Michalsen A, Knoblauch N, Tan L, et al Acupuncture in migraine: investigation of autonomic effects. Clin J Pain. 2008;24(2):106-15.

15. Shu Q, Wang H, Litscher D, Wu S, Chen L, Gaischek I, et al. Acupuncture and moxibustion have different effects on fatigue by regulating the autonomic nervous system: a pilot controlled clinical trial. Sci Rep. 2016;6:37846.

16. Lomuscio A, Belletti S, Battezzati PM, Lombardi F. Efficacy of acupuncture in preventing atrial fibrillation recurrences after electrical cardioversion. J Cardiovasc Electrophysiol. 2011;22(3):241-7.

17. Yin J, Yang M, Yu S, Fu H, Huang H, Yang B, et al. Effect of acupuncture at Neiguan point combined with amiodarone therapy on early recurrence after pulmonary vein electrical isolation in patients with persistent atrial fibrillation. J Cardiovasc Electrophysiol. 2019;30(6):910-7.

18. Chen LY, Chung MK, Allen LA, Ezekowitz M, Furie KL, McCabe $P$, et al. Atrial Fibrillation burden: moving beyond atrial fibrillation as a binary entity: a scientific statement from the American Heart Association. Circulation. 2018; 137(20).

19. Wang H, Du YH. Acupuncture and moxibustion. 3rd ed. Beijing (China): China Press of Traditional Chinese Medicine; 2012.

20. Shi XM, Liang FR. Science of acupuncture and moxibustion.1st ed. Beijing (China): China Press of Traditional Chinese Medicine; 2018.

21. Ma TT, Yu SY, Li Y, Liang FR, Tian XP, Zheng H, et al. Randomised clinical trial: an assessment of acupuncture on specific meridian or specific acupoint vs. sham acupuncture for treating functional dyspepsia. Aliment Pharmacol Ther. 2012;35(5):552-61.

22. Li Y, Zheng H, Witt CM, Roll S, Yu SG, Yan J, et al. Acupuncture for migraine prophylaxis: a randomized controlled trial. CMAJ. 2012;184(4):401-10.

23. Schulz KF, Altman DG, Moher D. CONSORT 2010 statement: updated guidelines for reporting parallel group randomized trials. Ann Intern Med. 2010;152(11):726-32.

24. MacPherson H, Altman DG, Hammerschlag R, Youping L, Taixiang W, White A, et al. Revised STandards for Reporting Interventions in Clinical Trials of Acupuncture (STRICTA): extending the CONSORT statement. PLoS Med. 2010;7(6):e1000261.

25. Chan AW, Tetzlaff JM, Gotzsche PC, Altman DG, Mann H, Berlin JA, et al. SPIRIT 2013 explanation and elaboration: guidance for protocols of clinical trials. BMJ. 2013;346:e7586.

26. Zhang JL. Treatment of 38 cases of chronic atrial fibrillation with Bushen Ningxin Decoction Combined with Acupuncture. Public Medical Forum Magazine. 2009;13(13):447-8.

27. Zhou QX, Liang Z. 65 cases of atrial fibrillation treated with acupuncture and medicine. J Emerg Tradit Chinese Med. 2000;9(S1):7.

28. Lim S. WHO standard acupuncture point locations. Evid Based Complement Alternat Med. 2010:7(2):167-8.
29. Cao KJ, Chen KP, Chen ML, Gao LJ, Hua W, Huang H, et al. Atrial fibrillation: current understanding and treatment recommendations-2018. Chinese J Cardiac Pacing Electrophysiol. 2018;32(4):315-68.

30. Spertus J, Dorian P, Bubien R, Lewis S, Godejohn D, Reynolds MR, et al. Development and validation of the Atrial Fibrillation Effect on QualiTy-ofLife (AFEQT) Questionnaire in patients with atrial fibrillation. Circ Arrhythm Electrophysiol. 2011;4(1):15-25.

31. Devilly GJ, Borkovec TD. Psychometric properties of the credibility/ expectancy questionnaire. J Behav Ther Exp Psychiatry. 2000;31(2):73-86.

32. Kieser M, Wassmer G. On the use of the upper confidence limit for the variance from a pilot sample for sample size determination. Biom J. 1996; 38(8):941-9.

33. Mark DB, Anstrom KJ, Sheng S, Piccini JP, Baloch KN, Monahan KH, et al. Effect of catheter ablation vs medical therapy on quality of life among patients with atrial fibrillation. JAMA. 2019;321(13):1275.

34. Blomström-Lundqvist C, Gizurarson S, Schwieler J, Jensen SM, Bergfeldt L, Kennebäck $G$, et al. Effect of catheter ablation vs antiarrhythmic medication on quality of life in patients with atrial fibrillation. JAMA. 2019;321(11):1059.

35. Chen ZX, Li Y, Zhang XG, Chen S, Yang WT, Zheng XW, et al. Sham electroacupuncture methods in randomized controlled trials. Sci Rep. 2017; 7:40837.

36. Tan J, Suen LKP, Wang T, Molassiotis A. Sham acupressure controls used in randomized controlled trials: a systematic review and critique. PLoS One. 2015;10(7):e132989.

37. Yang JW, Wang LQ, Zou X, Yan SY, Wang Y, Zhao JJ, et al. Effect of acupuncture for postprandial distress syndrome: a randomized clinical trial. Ann Intern Med. 2020;172(12):777-85.

38. Liu ZS, Yan SY, Wu JN, He LY, Li N, Dong GR, et al. Acupuncture for chronic severe functional constipation: a randomized trial. Ann Intern Med. 2016; 165(11):761-9.

\section{Publisher's Note}

Springer Nature remains neutral with regard to jurisdictional claims in published maps and institutional affiliations.

\section{Ready to submit your research? Choose BMC and benefit from:}

- fast, convenient online submission

- thorough peer review by experienced researchers in your field

- rapid publication on acceptance

- support for research data, including large and complex data types

- gold Open Access which fosters wider collaboration and increased citations

- maximum visibility for your research: over 100M website views per year

At BMC, research is always in progress.

Learn more biomedcentral.com/submissions 